\title{
Editorial
}

\section{Astronomical Massive Data Processing Technology}

\author{
Feng Wang $\mathbb{D}^{1},{ }^{1}$ Wenda Cao, ${ }^{2}$ Denis Bastieri, ${ }^{3}$ Junhui Fan, ${ }^{1}$ and Chenzhou Cui ${ }^{4}$ \\ ${ }^{1}$ Center for Astrophysics, Guangzhou University, Guangzhou, China \\ ${ }^{2}$ Big Bear Solar Observatory, Big Bear, USA \\ ${ }^{3}$ Padova University, Padova, Italy \\ ${ }^{4}$ National Astronomical Observatory, Chinese Academy of Sciences, Beijing, China \\ Correspondence should be addressed to Feng Wang; fengwang@gzhu.edu.cn
}

Received 1 April 2020; Accepted 1 April 2020; Published 1 May 2020

Copyright (c) 2020 Feng Wang et al. This is an open access article distributed under the Creative Commons Attribution License, which permits unrestricted use, distribution, and reproduction in any medium, provided the original work is properly cited.

Astronomy has become one of the biggest consumers of computing resources in the past 10 years. New computational techniques are emerging and dedicated to the various fields of astronomy research. Thus, we can already see such a development trend that astronomical studies will become larger in all dimensions: larger data collections, more accurate data analysis and processing, and more detailed results. The significant increase of these large-size astronomical studies performed by using instruments built around the world requires not only huge processing power but also clever system design, all the way from hardware construction to software development and deployment. With this special issue, we want to cover most relevant aspects related to new computing technology to illustrate the readers the wide scope opened by these observational techniques in computing astronomy.

There are total 6 papers collected in this special issue, which concerned massive astronomical data archive and management, data analysis, visualization and application, and data reduction in modern telescope. In what follows, we present the main contents of this special issue split by subject:

Two papers of this issue are devoted to massive data archive and management techniques by $\mathrm{H}$. Zhang et al. and X. Ye et al. H. Zhang et al. presented a robust way to transfer massive data. More than 84,000 pulsar observation data files in the PSRFITS format have been released with a maximum file size of 1 GB. X. Ye et al. designed effective mechanisms to ensure the high stability of the system and to control the data transmission when the network is unstable through adaptive algorithms which could transfer the massive data from observatory to its data processing center.

Four papers discussed the massive data analysis, which showed how to apply information processing techniques in current astronomical data processing. S. Shrestha and D.-Y. Choi proposed a technique that provides sufficiently accurate estimation for $\mathrm{Ku}$-band signal attenuation in site specifically whose effectiveness is performed through the statistical analysis against the established rain attenuation models.

In the study of W. Wang and Y. Yan, a new restoration method for solar radio image was proposed. Two images with different weighting functions from the same data are combined. This restoration method has been applied to data processing of Mingantu spectral radioheliograph. M. Zhu et al. extracted filaments based on morphology component analysis from radio astronomical images. The experimental results showed the method proposed by the authors is effective and final images have higher peak signal-to-noise ratio (PSNR). Z. Qiang et al. applied an adaptive background learning method to detect the CMEs (coronal mass ejections). Comparing the result with CDAW, CACTus, SEEDS, and CORIMP catalogs, the new method detected about $73 \%$ of the CMEs listed in the CDAW CME catalog, which is identified by human visual inspection. 


\title{
Conflicts of Interest
}

All authors declare that there are no conflicts of interest regarding the publication of this article.

\author{
Feng Wang \\ Wenda Cao \\ Denis Bastieri \\ Junhui Fan \\ Chenzhou Cui
}

